\title{
Effective hadronic Lagrangian for charm mesons
}

\author{
Ziwei Lin and C.M. Ko \\ Cyclotron Institute and Physics Department, Texas A\& M University, College \\ Station, TX 77843-3366, USA
}

\begin{abstract}
An effective hadronic Lagrangian including the charm mesons is introduced to study their interactions in hadronic matter. Using coupling constants that are determined either empirically or by the SU(4) symmetry, we have evaluated the absorption cross sections of $J / \psi$ and the scattering cross sections of $D$ and $D^{*}$ by $\pi$ and $\rho$ mesons.
\end{abstract}

\section{Introduction}

Suppression of charmonium production in high energy heavy ion collisions is a possible signature for the quark-gluon plasma (QGP) formed in ultra-relativistic heavy ion collisions [1]. Recent data from the $\mathrm{Pb}+\mathrm{Pb}$ collision at $P_{\text {lab }}=158 \mathrm{GeV} / \mathrm{c}$ in the NA50 experiment at CERN [2] have shown an anomalously large $J / \psi$ suppression in high $E_{\mathrm{T}}$ events. While there are suggestions that this anomalous suppression may be due to the formation of the QGP [3, 4 , more conventional mechanisms based on $J / \psi$ absorption by comoving hadrons have also been proposed as a possible explanation [5, 6. Since the latter scenario depends on the value of $J / \psi$ absorption cross sections by hadrons, it is important to have better knowledge of these cross sections in order to understand the observed anomalous charmonium suppression [7]. Even in heavy ion collisions at the Relativistic Heavy Ion Collider (RHIC), where the QGP is most likely to be formed, the effect of hadronic absorption of $J / \psi$ is still non-negligible [8]. Furthermore, one needs to know if $J / \psi$ can also be produced from the hot hadronic matter in the later stage of heavy ion collisions [9, 10].

Also, charm mesons such as $D$ and $D^{*}$ are expected to be abundantly produced in heavy ion collisions at RHIC energies and beyond. It has been shown that the high mass $(M>2 \mathrm{GeV})$ dilepton spectrum at RHIC may be dominated by decays from charm and bottom hadrons 11]. Since charm quarks may lose appreciable energies in a quark-gluon plasma via gluon radiations, study of charm meson spectrum in heavy ion collisions could provide useful information on the properties of the QGP [12, 13]. However, charm mesons may interact strongly with hadrons during later stage of heavy ion collisions, leading to possible changes in their final spectra. As a result, the dilepton spectrum from the decay of charm meson pairs could also be modified [14]. To study the energy loss of charm quarks in the QGP thus requires the understanding of charm meson interactions in hadronic matter.

Various approaches have been used in evaluating the charmonium absorption cross sections by hadrons. In the quark-exchange model, an earlier study 15 has shown that the $J / \psi$ absorption cross section by pion, $\sigma_{\pi \psi}$, has a peak value of about $7 \mathrm{mb}$ at $E_{\text {kin }} \equiv \sqrt{s}-m_{\pi}-m_{\psi} \simeq 0.8 \mathrm{GeV}$, but a recent study [16] gives a peak value of 
only $\sigma_{\pi \psi} \sim 1 \mathrm{mb}$ at the same $E_{\text {kin }}$ region. On the other hand, the perturbative QCD approach 17] predicts that the $J / \psi$ dissociation cross section increases monotonously with $E_{\text {kin }}$ and has a value of only about $0.1 \mathrm{mb}$ around $E_{\text {kin }} \sim 0.8 \mathrm{GeV}$. Charmonium absorption cross sections by hadrons have also been studied in meson-exchange models based on effective hadronic Lagrangians. Using pseudoscalar-pseudoscalar-vectormeson (PPV) couplings and without form factors at the interaction vertices, Matinyan and Müller [18] have found $\sigma_{\pi \psi} \simeq 0.3 \mathrm{mb}$ at $E_{\text {kin }}=0.8 \mathrm{GeV}$. In a recent study, Haglin 19 has included also the three-vector-meson (VVV) and four-point couplings (or contact terms), and obtained much larger values for the $J / \psi$ absorption cross sections. Large discrepancies in the magnitude of $\sigma_{\pi \psi}$ (as well as $\sigma_{\rho \psi}$ ) thus exist among the predictions from these three approaches. In this study, we use a mesonexchange model as in [19, 20] but treat differently the VVV and four-point couplings in the effective Lagrangian. For charm meson scattering cross sections with pion and rho meson, previous studies from the meson-exchange model include only the PPV interactions [21]. We now also use the extended hadronic Lagrangian to study these cross sections.

\section{The effective Lagrangian}

The free Lagrangian for pseudoscalar and vector mesons in the limit of $\mathrm{SU}(4)$ invariance can be written as $\mathcal{L}_{0}=\operatorname{Tr}\left(\partial_{\mu} P^{\dagger} \partial^{\mu} P\right)-\operatorname{Tr}\left(F_{\mu \nu}^{\dagger} F^{\mu \nu}\right) / 2$, where $F_{\mu \nu}=\partial_{\mu} V_{\nu}-\partial_{\nu} V_{\mu}$, and $P$ and $V$ denote, respectively, the $4 \times 4$ pseudoscalar and vector meson matrices in SU(4) 21]. To obtain the couplings between pseudoscalar and vector mesons, we introduce the minimal substitution

$$
\partial_{\mu} P \rightarrow \mathcal{D}_{\mu} P=\partial_{\mu} P-\frac{i g}{2}\left[V_{\mu} P\right], F_{\mu \nu} \rightarrow \partial_{\mu} V_{\nu}-\partial_{\nu} V_{\mu}-\frac{i g}{2}\left[V_{\mu}, V_{\nu}\right] .
$$

The effective Lagrangian is then given by

$$
\begin{aligned}
\mathcal{L} & =\mathcal{L}_{0}+i g \operatorname{Tr}\left(\partial^{\mu} P\left[P, V_{\mu}\right]\right)-\frac{g^{2}}{4} \operatorname{Tr}\left(\left[P, V_{\mu}\right]^{2}\right) \\
& +i g \operatorname{Tr}\left(\partial^{\mu} V^{\nu}\left[V_{\mu}, V_{\nu}\right]\right)+\frac{g^{2}}{8} \operatorname{Tr}\left(\left[V_{\mu}, V_{\nu}\right]^{2}\right) .
\end{aligned}
$$

Since the SU(4) symmetry is explicitly broken by hadron masses, terms involving hadron masses are added to Eq.(2) using the experimentally determined values.

The effective Lagrangian in Eq. (2) is generated by minimal substitution, which is equivalent to treating vector mesons as gauge particles. The gauge invariance leads to the current conservation; i.e., in the limit of zero vector meson masses, degenerate pseudoscalar meson masses, and $\mathrm{SU}(4)$ invariant coupling constants, one has $\mathcal{M}^{\lambda_{k} \ldots \lambda_{l}} p_{j \lambda_{j}}=0$ for any given process, where the index $\lambda_{j}$ denotes the external vector meson $j$. We have checked that all the amplitudes without form factors satisfy the requirement of current conservation.

The above effective Lagrangian allows us to study various interactions of charm mesons and $J / \psi$ with hadrons. These include the charm meson scattering such as $\pi D \leftrightarrow \rho D^{*}$, the charm meson production and annihilation such as $\pi \pi \leftrightarrow D \bar{D}$, and the charmonium absorption and annihilation such as $\pi \psi \leftrightarrow D \bar{D}^{*}$. This effective hadronic Lagrangian has also been extended to SU(5) to study $\Upsilon$ absorption in hadronic matter [22]. In the following, we show the results for $J / \psi$ absorption and charm meson scattering by pion and rho meson. 

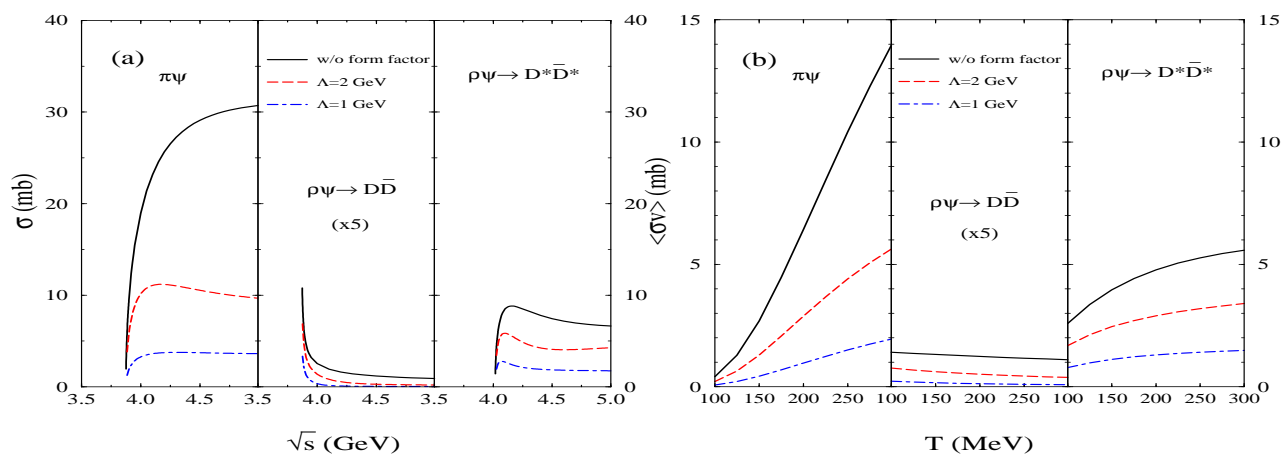

Figure 1. (a) $J / \psi$ absorption cross sections as functions of energy. (b) Thermalaveraged $J / \psi$ absorption cross sections as functions of temperature.

\section{3. $J / \psi$ absorptions}

For $J / \psi$ absorption by $\pi$ and $\rho$ mesons, we study the following processes:

$$
\pi \psi \rightarrow D^{*} \bar{D}, \pi \psi \rightarrow D \bar{D}^{*}, \rho \psi \rightarrow D \bar{D}, \rho \psi \rightarrow D^{*} \bar{D}^{*} .
$$

The full amplitudes for these processes can be found in [23].

From the $D^{*}$ decay width [24, the coupling constant $g_{\pi D D^{*}}$ is found to be $g_{\pi D D^{*}}=4.4$. Using the vector meson dominance (VMD) model, we determine other three-point coupling constants as 23]

$$
g_{\rho D D}=g_{\rho D^{*} D^{*}}=2.52, g_{\psi D D}=g_{\psi D^{*} D^{*}}=7.64 \text {. }
$$

For the four-point coupling constants, there is no empirical information, and we thus use the $\mathrm{SU}(4)$ relations to determine their values as

$$
g_{\pi \psi D D^{*}}=g_{\pi D D^{*}} g_{\psi D D}, g_{\rho \psi D D}=2 g_{\rho D D} g_{\psi D D}, g_{\rho \psi D^{*} D^{*}}=g_{\rho D^{*} D^{*}} g_{\psi D^{*} D^{*}} .
$$

Form factors are introduced at interaction vertices to take into account the composite nature of hadrons. Unfortunately, there is no empirical information on form factors involving charmoniums and charm mesons. We thus take the form factors as the usual monopole form at the three-point $t$ and $u$ channel vertices, i.e., $f_{3}(t$ or $u)=\Lambda^{2} /\left(\Lambda^{2}+\mathbf{q}^{2}\right)$, where $\Lambda$ is a cutoff parameter, and $\mathbf{q}^{2}$ is the squared three momentum transfer in the c.m. frame, given by $\left(\mathbf{p}_{\mathbf{1}}-\mathbf{p}_{\mathbf{3}}\right)_{\text {c.m. }}^{2}$ and $\left(\mathbf{p}_{\mathbf{1}}-\mathbf{p}_{\mathbf{4}}\right)_{\text {c.m. }}^{2}$ for $t$ and $u$ channel processes, respectively. For simplicity, we use the same value for all cutoff parameters, i.e., $\Lambda_{\pi D D^{*}}=\Lambda_{\rho D D}=\Lambda_{\rho D^{*} D^{*}}=\Lambda_{\psi D D}=\Lambda_{\psi D^{*} D^{*}} \equiv \Lambda$, and choose $\Lambda$ as either 1 or $2 \mathrm{GeV}$ to study the uncertainties due to form factors. We also assume that the form factor at four-point vertices is given by $f_{4}=\left[\Lambda^{2} /\left(\Lambda^{2}+\overline{\mathbf{q}}^{2}\right)\right]^{2}$, where $\overline{\mathbf{q}^{2}}$ is the average value of the squared three momentum transfers in $t$ and $u$ channels, $\overline{\mathbf{q}^{2}}=p_{i, \text { c.m. }}^{2}+p_{f, \text { c.m. }}^{2}$.

Figure 1a shows the cross section as a function of the c.m. energy of initialstate mesons. Although form factors strongly suppress the cross sections, the $J / \psi$ absorption cross sections remain appreciable after including form factors. The values for $\sigma_{\pi \psi}$ and $\sigma_{\rho \psi}$ are roughly 7 and $3 \mathrm{mb}$, respectively, and are comparable to those used in phenomenological studies of $J / \psi$ absorption by comoving hadrons in relativistic heavy ion collisions [5, 6, 25]. The thermal average of $J / \psi$ absorption cross sections is shown in figure 1b. At the temperature of $150 \mathrm{MeV}$, for example, $\left\langle\sigma_{\pi \psi} v\right\rangle$ and $\left\langle\sigma_{\rho \psi} v\right\rangle$ are about 1 and $2 \mathrm{mb}$, respectively. 

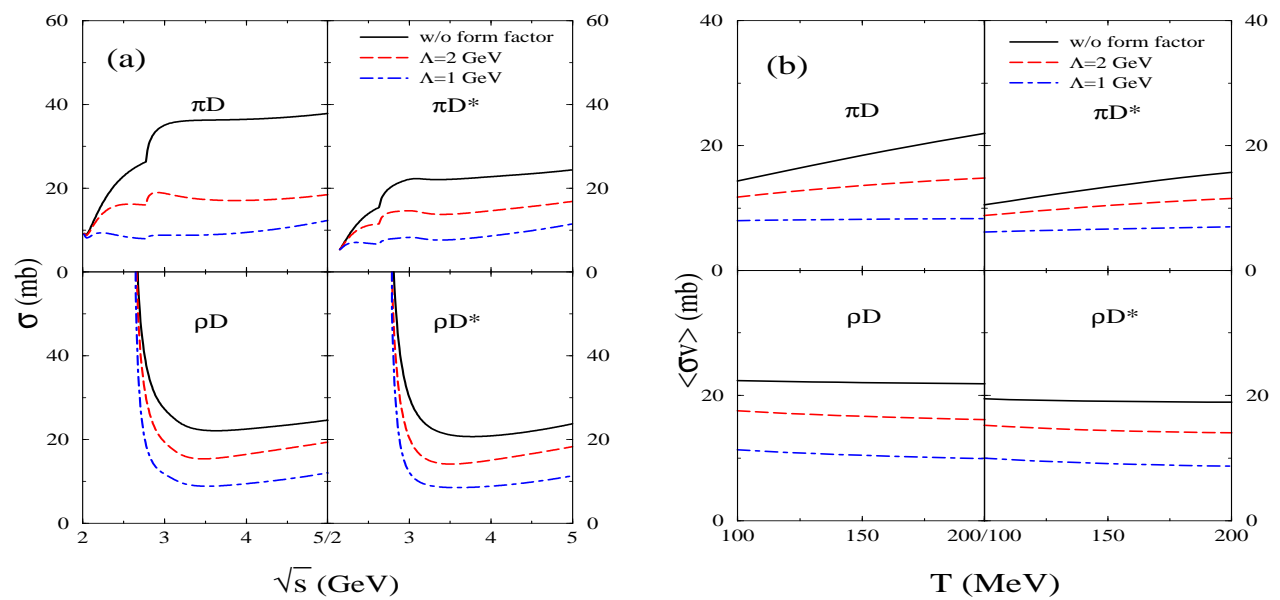

Figure 2. (a) Scattering cross sections of $D$ and $D^{*}$ as functions of energy. (b) Thermal-averaged cross sections as functions of temperature.

\section{Charmed meson scattering}

We consider the following processes for charm meson scattering by $\pi$ and $\rho$ mesons:

$$
\pi D \leftrightarrow \rho D^{*}, \pi D \rightarrow \pi D, \pi D^{*} \rightarrow \pi D^{*}, \pi D^{*} \leftrightarrow \rho D, \rho D \rightarrow \rho D, \rho D^{*} \rightarrow \rho D^{*} .
$$

There are also similar processes for anticharm mesons. The full amplitudes for the above processes can be found in 26 .

For the coupling constants, we use $g_{\rho \pi \pi}=6.1$ [27]. The SU(4) symmetry then gives $g_{\rho \rho \rho}=g_{\rho \pi \pi}$ and the following relations for the four-point couplings:

$g_{\pi \rho D D^{*}}=g_{\pi D D^{*}} g_{\rho D D}, g_{\pi \pi D^{*} D^{*}}=2 g_{\pi D D^{*}}^{2}, g_{\rho \rho D D}=2 g_{\rho D D}^{2}, g_{\rho \rho D^{*} D^{*}}=g_{\rho D^{*} D^{*}}^{2}$. (5)

Form factors at the $s$ channel vertices are taken as $f_{3}(s)=\Lambda^{2} /\left(\Lambda^{2}+\mathbf{k}^{2}\right)$ [28], with $\mathbf{k}$ denoting the three momentum of either the incoming or outgoing particles in the c.m. frame, i.e., $\mathbf{k}^{2}=p_{i, \text { c.m. }}^{2}$ or $p_{f, \text { c.m. }}^{2}$. For form factors at four-point vertices, we take them to be $f_{4}^{\prime}=\left[\Lambda^{2} /\left(\Lambda^{2}+\overline{\mathbf{k}^{2}}\right)\right]^{2}$, where $\overline{\mathbf{k}^{2}}$ is the average value of the squared three momenta in the form factors for the $s, t$, and $u$ channels, i.e., $\overline{\mathbf{k}}^{2}=5\left(p_{i, \text { c.m. }}^{2}+p_{f, \text { c.m. }}^{2}\right) / 6$.

The charm meson scattering cross sections and their thermal averages are shown in figure $2 \mathrm{a}$ and $2 \mathrm{~b}$, respectively. As expected, the magnitude of the cross sections decreases with decreasing cutoff parameter. For the cutoff parameters used here, the cross sections for $\pi D, \pi D^{*}, \rho D$ and $\rho D^{*}$ scattering are all roughly between 10 and $20 \mathrm{mb}$. We note that form factors only suppress modestly (by a factor of two and less) these total cross sections and their thermal averages. This is due to the fact that these cross sections are dominated by elastic processes, which involve small momentum transfer near the threshold. In contrast, the process $\pi \psi \rightarrow D^{*} \bar{D}$ has a large threshold, and form factors suppress its cross section by as much as a factor of 8 .

\section{Summary}

In summary, we have introduced an effective hadronic Lagrangian, that includes the charm mesons, to study the interactions of charmed mesons and $J / \psi$ in hadronic 
matter. In particular, we have calculated the absorption cross sections of $J / \psi$ and scattering cross sections of charmed mesons by $\pi$ and $\rho$ mesons. We find that the $J / \psi$ absorption cross sections are much larger than those in a previous study, where only pseudoscalar-pseudoscalar-vector-meson couplings were considered. Including form factors at the interaction vertices, the values for $\sigma_{\pi \psi}$ and $\sigma_{\rho \psi}$ are about $7 \mathrm{mb}$ and $3 \mathrm{mb}$, respectively, and their thermal averages at the temperature of $150 \mathrm{MeV}$ are roughly $1 \mathrm{mb}$ and $2 \mathrm{mb}$, respectively. These values suggest that the absorption of $J / \psi$ by comoving hadrons may play an important role in $J / \psi$ suppression in relativistic heavy ion collisions. We also find that the scattering cross sections of $D$ or $D^{*}$ by $\pi$ or $\rho$ mesons are all about $10 \mathrm{mb}$ and thus expect these scatterings to significantly modify the charm meson spectra in heavy ion collisions.

\section{Acknowledgments}

This work was supported in part by the National Science Foundation under Grant No. PHY-9870038, the Welch Foundation under Grant No. A-1358, and the Texas Advanced Research Program under Grant No. FY99-010366-0081.

\section{References}

[1] Matsui T and Satz H 1986 Phys. Lett. B 178416.

[2] Gonin M et al (NA50 Collaboration) 1996 Nucl. Phys. A 610 404c; Abreu M C et al (NA50 Collaboration) 1989 Phys. Lett. B 450456.

[3] Blaizot J P and Ollitrault J Y 1996 Phys. Rev. Lett. 771703.

[4] Wong C Y 1998 Nucl. Phys. A 630487.

[5] Cassing W and Ko C M 1997 Phys. Lett. B 396 39; Cassing W and Bratkovskaya E L 1997 Nucl. Phys. A 623570.

[6] Armesto N and Capella A 1998 Phys. Lett. B 43023.

[7] For recent reviews, see, e.g., Vogt R 1999 Phys. Rep. 310 197; Satz H 2000 Rept. Prog. Phys. 631511.

[8] Zhang B, Ko C M, Li B A, Lin Z and Sa B H 2000 Preprint nucl-th/0007003.

[9] Ko C M, Wang X N, Zhang B and Zhang X F 1998 Phys. Lett. B 444237.

[10] Braun-Munzinger B and Redlich K 1999 Nucl. Phys. A 661546.

[11] Vogt R, Jacak B V, McGaughey P L and Ruuskanen P V 1994 Phys. Rev. D 493345.

[12] Shuryak E 1997 Phys. Rev. C 55961.

[13] Lin Z, Vogt R and Wang X N 1998 Phys. Rev. C 57899.

[14] Lin Z and Wang X N 1998 Phys. Lett. B 444245.

[15] Martins K, Blaschke D and Quack E 1995 Phys. Rev. C 512723.

[16] Wong C Y, Swanson E S and Barnes T 1999 Preprint hep-ph/9912431.

[17] Kharzeev D and Satz H 1994 Phys. Lett. B 334155.

[18] Matinyan S G and Müller B 1998 Phys. Rev. C 582994.

[19] Haglin K 2000 Phys. Rev. C 61031902.

[20] Haglin K and Gale C 2000 Preprint nucl-th/0002029.

[21] Lin Z, Ko C M and Zhang B 2000 Phys. Rev. C 61024904.

[22] Lin Z and Ko C M 2000 Preprint nucl-th/0007027.

[23] Lin Z and Ko C M 2000 Phys. Rev. C 62034903.

[24] Colangelo P, De Fazio F and Nardulli G 1994 Phys. Lett. B 334 175; Belyaev V M, Braun V M, Khodjamirian A and Ruckl R 1995 Phys. Rev. D 516177.

[25] Kahana D E and Kahana S H 1999 Phys. Rev. C 59 1651; Sa B H, Tai A, Wang H and Liu F H 1999 Phys. Rev. C 59 2728; Spieles C, Vogt R, Gerland L, Bass S A, Bleicher M, Stöcker $\mathrm{H}$ and Greiner W 1999 Phys. Rev. C 60054901.

[26] Lin Z, Di T G and Ko C M 2000 Preprint nucl-th/0006086.

[27] Brown G E, Ko C M, Wu Z G and Xia L H 1991 Phys. Rev. C 43 1881; Ko C M and Seibert D 1994 Phys. Rev. C 492198.

[28] Tsushima K, Huang S W and Faessler A 1994 Phys. Lett. B 337 245; Bratkovskaya E L and Ko C M 1999 Phys. Lett. B 445265. 